\title{
Vierdimensionale Mineralerkennung mittels hyperspektraler Fluoreszenzbilder
}

\author{
Sebastian Bauer und Fernando Puente León \\ Institut für Industrielle Informationstechnik (IIIT), Karlsruher Institut für Technologie (KIT) \\ Hertzstraße 16, Geb. 6.35, 76187 Karlsruhe
}

\section{Zusammenfassung}

Die zuverlässige automatisierte Unterscheidung von Mineralen ist Voraussetzung für den erfolgreichen Einsatz von Mineralsortiermaschinen im industriellen Umfeld. Wünschenswert ist eine berührungslose, zerstörungsfreie und schnelle Erfassung von Materialeigenschaften sowie eine einfache nachfolgende Klassifikation. Für die Erfassung der Materialeigenschaften bieten sich optische Technologien an. Da sich viele Minerale aufgrund von farblicher Ähnlichkeit anhand von Farbbildern nicht unterscheiden lassen, basiert ein von uns kürzlich vorgeschlagener Alternativansatz auf der Gewinnung von hyperspektralen Fluoreszenzbildern. Durch Variation der Anregungswellenlänge ergeben sich vierdimensionale Signale. Diese Signale werden fusioniert und Merkmale zur einfachen Klassifikation abgeleitet.

Keywords: Fluoreszenz, Hyperspektralbild, Bildverarbeitung, Mineralerkennung, Salienzkarten.

\section{Einleitung}

Die automatisierte Sortierung von Mineralen erfordert die zuverlässige Bestimmung von Materialparametern, anhand derer sich unterschiedliche Mineralsorten unterscheiden lassen. Optische Verfahren stellen eine berührungslose und schnelle Möglichkeit zur Feststellung solcher Materialparameter dar. Da sich allerdings viele Minerale aufgrund von farblicher Ähnlichkeit mit bloßem Auge kaum unterscheiden lassen, scheiden herkömmliche Farbbilder als Basis für die Unterscheidung aus. Stattdessen werden hyperspektrale Bilder aufgezeichnet, die statt drei breitbandigen Farbkanälen bis zu mehreren hundert, nur wenige Nanometer breite Kanäle besitzen. Somit steht pro Pixel ein vollständiges Spektrum zur Verfügung. Solche Bilder können nicht nur im für den Menschen sichtbaren Wellenlängenbereich aufgezeichnet werden, sondern auch im Nahinfrarot-Wellenlängenbereich von 780 bis $2500 \mathrm{~nm}$ [1]. In diesem Bereich ist die Reflektanz von chemischen Materialeigenschaften geprägt, wodurch sich diskriminative spektrale Signaturen der Minerale ergeben. Andererseits ist die Gewinnung solcher Hyperspektralbilder relativ aufwändig, da für Detektoren im Nahinfrarot Silizium als Halbleitermaterial ausscheidet und deshalb auf teurere, weniger etablierte Materialien zurückgegriffen werden muss. Auch ist das thermische Rauschniveau von Nahinfrarotkameras natur- gemäß deutlich höher als das von Kameras im sichtbaren Wellenlängenbereich.

Ein von uns kürzlich vorgeschlagener Alternativansatz $[2,3]$ basiert nicht auf der Gewinnung von hyperspektralen Reflektanz-, sondern Fluoreszenzbildern. Dabei wird nicht im Wellenlängenbereich, in dem die Aufnahme stattfindet, beleuchtet, sondern mit energiereichem ultraviolettem Licht (UV-Licht). Durch die Anregung mit UV-Licht werden Elektronen angeregt und fallen nach einer gewissen Zeit wieder in ihren Grundzustand zurück. Dabei werden Fluoreszenzphotonen ausgesendet, die geringere Energie als die anregenden Photonen besitzen. Damit liegt die emittierte Strahlung im sichtbaren Wellenlängenbereich statt im Nahinfrarot und kann mit herkömmlichen Siliziumdetektoren erfasst werden. Vorteil dieser Methode ist die einfachere Bildgewinnung. Nachteil ist, dass viele Minerale nur schwach fluoreszieren, sodass gegebenenfalls starke UVLichtquellen verwendet werden müssen. AuBerdem ist die spektrale Zusammensetzung der Fluoreszenz stark vom Gehalt an sogenannten Aktivatoren (beispielsweise Gitterfehlstellen oder Lanthanoide) [4] abhängig. Aus diesem Grund können Minerale gleicher Sorte, die aus unterschiedlichen Lagerstätten stammen, stark unterschiedliche Fluoreszenzspektren aufweisen.

Da auch unterschiedliche Mineralsorten teilweise recht ähnliche Spektren aufweisen, wird im Rahmen dieser Veröffentlichung untersucht, 

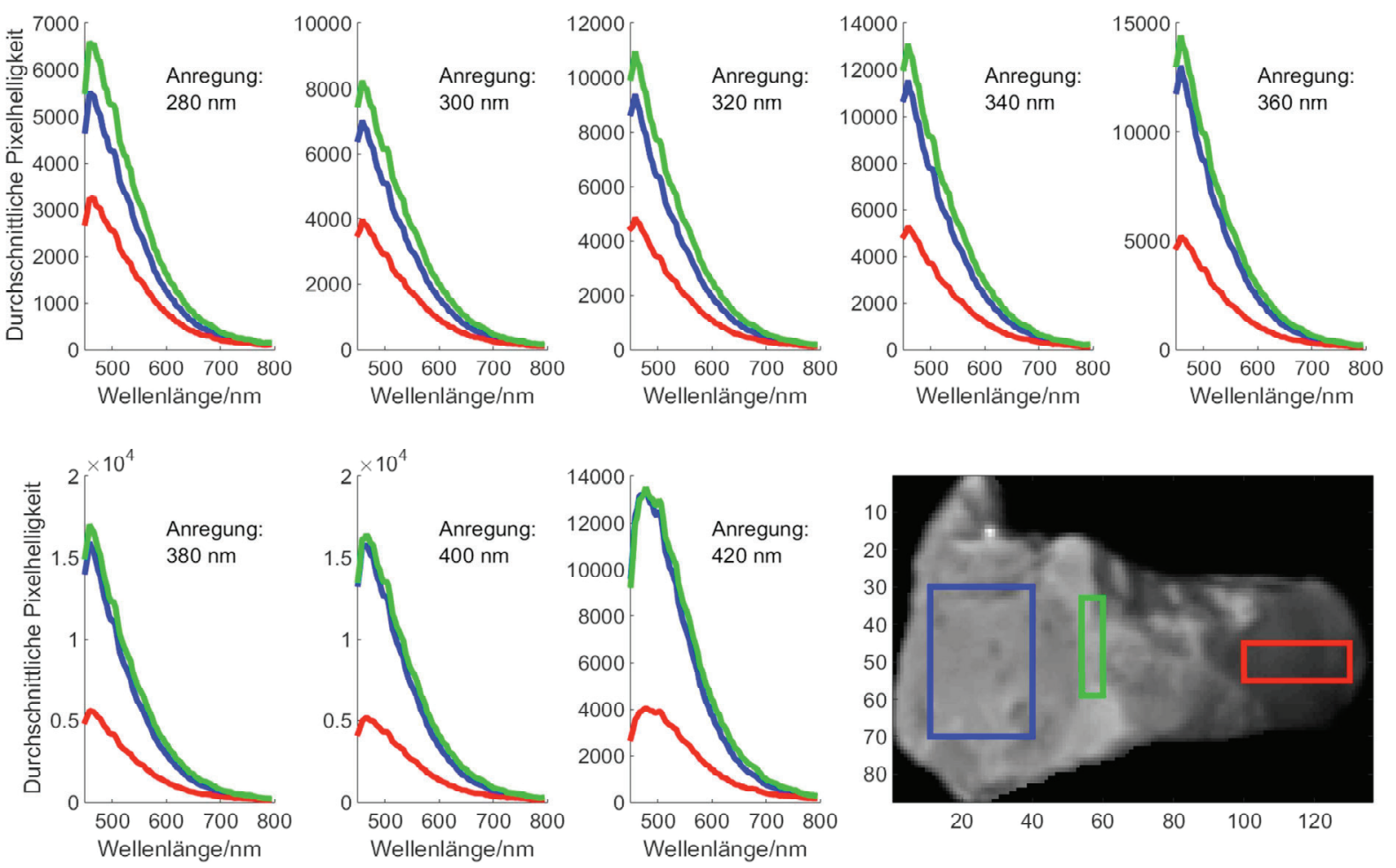

Abbildung 1: Örtliche Abhängigkeit der Fluoreszenzspektren einer Mineralprobe bei verschiedenen Anregungswellenlängen. Es fällt auf, dass sich das Intensitätsverhältnis zwischen blauer/grüner und roter Region deutlich ändert: Bei $280 \mathrm{~nm}$ Anregungswellenlänge beträgt das Verhältnis etwa 2, bei 420 nm Anregungswellenlänge 3.

inwiefern die Verwendung von Hyperspektralbildern bei mehreren Anregungswellenlängen gewinnbringend für die Klassifikation eingesetzt werden kann. Dabei wird ausgenutzt, dass die spektrale Zusammensetzung des Fluoreszenzlichts auch abhängig von der Anregungswellenlänge ist, siehe Abbildung 1. Bei jeder Anregungswellenlänge liegt ein kompletter dreidimensionaler Datensatz (der sogenannte Datenwürfel) vor, womit die Anregungswellenlänge eine vierte Dimension darstellt.

Die in der vierdimensionalen Datenstruktur enthaltene Information soll analysiert und fusioniert werden. Wünschenswert ist dabei, dass durch die Fusion dieser vier Dimensionen ein einzelnes dreidimensionales Hyperspektralbild resultiert, das eine erfolgreichere Unterscheidung als die einzelnen Hyperspektralbilder bei den verschiedenen Anregungswellenlängen erlaubt.

Eine Möglichkeit ist es, für die Fusion Salienzkarten zu nutzen. Diese werden von mathematischen Verfahren erstellt, die das visuelle Empfinden des Menschen nachbilden. Ziel ist es, in Bildern automatisiert diejenigen Bereiche zu identifizieren, auf die sich die Aufmerksamkeit des Menschen nach Beginn der Betrachtung sehr schnell konzentriert. In Abbildung 1 fällt neben der Abhängigkeit der Fluoreszenzspektren von der Anregungswellenlänge auBerdem auf, dass sich das Intensitätsverhältnis in manchen Regionen ebenfalls in Abhängigkeit der Anregungswellenlänge ändert. Den Verfahren zur Salienzbestimmung kommt daher bei der Fusion unter anderem die Aufgabe zu, zu bestimmen, welche Bildregionen sich anders verhalten als andere. Das Prinzip der Salienzbestimmung wird nach der jetzt folgenden Erklärung der Messung noch genauer beschrieben werden.

\section{Messaufbau und Messprinzip}

Aus dem kontinuierlichen Spektrum einer $300 \mathrm{~W}$-Xenonlampe werden mittels eines Monochromators bestimmte Wellenlängenbereiche ausgeschnitten. Im vorliegenden Fall wird die Anregungswellenlänge im Bereich von 280$420 \mathrm{~nm}$ in Schritten von $20 \mathrm{~nm}$ variiert; die Halbwertsbreite beträgt jeweils $15 \mathrm{~nm}$. Mittels eines Aluminiumspiegels werden die Lichtstrahlen auf die Probe gelenkt. Das Fluoreszenzlicht wird durch ein akustooptisches Filter (acousto-optical tunable filter, AOTF) vom Typ Gooch\&Housego HSi-300 spektral gefiltert und mit einer Andor iXon $_{3} 897$ EMCCD-Kamera (electron multiplying charge-coupled device) erfasst. Dabei wird der Wellenlängenbereich von 450-790 nm im Abstand von $4 \mathrm{~nm}$ abgetastet, sodass sich insgesamt pro Anregung 86 zweidimensionale Bilder der Szene ergeben; bei jeder Aufzeichnungswellenlänge eines. Die Belichtungszeit wird dabei so gewählt, dass die 
Szene bei jeder Anregungswellenlänge ungefähr mit derselben Anzahl von Photonen belichtet wird. Der gesamte Vorgang spielt sich in einem abgedunkelten Kasten ab. Da, wie eingangs erwähnt, die Fluoreszenz bei manchen Mineralen relativ lichtschwach ausgeprägt ist, werden bei diesen nur wenige Photonen pro Pixel erfasst, weshalb die entstandenen Bilder mit einem speziellen Verfahren entrauscht werden. In Bezug auf die schwache Lichtintensität ist zu beachten, dass die Szene durch die spektrale Begrenzung der Anregungswellenlänge und andere Effekte lediglich mit einer Strahlungsleistung von weniger als einem Watt angeregt wird.

Insgesamt werden 82 Minerale aus 9 verschiedenen Sorten untersucht.

\section{Quaternionen und Methoden zur Salienzbe- stimmung}

Um die Methoden zur Salienzbestimmung genauer erläutern zu können, sollen zunächst Quaternionen vorgestellt werden. Quaternionen sind vereinfacht gesagt vierdimensionale komplexe Zahlen mit drei Imaginärteilen. Eine Quaternion $q$ lässt sich schreiben als

$q=w+\mathrm{i} \cdot x+\mathrm{j} \cdot y+\mathrm{k} \cdot z$,

wobei $\mathrm{i}, \mathrm{j}$ und $\mathrm{k}$ Basiselemente darstellen. Für diese gilt

$\mathrm{i}^{2}=\mathrm{j}^{2}=\mathrm{k}^{2}=\mathrm{ijk}=-1$,

$\mathrm{ij}=\mathrm{k}, \quad \mathrm{jk}=\mathrm{i}, \quad \mathrm{ki}=\mathrm{j}$,

$\mathrm{ji}=-\mathrm{k}, \quad \mathrm{kj}=-\mathrm{i}, \quad \mathrm{ik}=-\mathrm{j}$.

Analog zu den komplexen Zahlen besitzen Quaternionen einen Realteil $\mathfrak{R}(q)=W$ und einen Imaginärteil $\mathfrak{I}(q)=\mathrm{i} \cdot x+\mathrm{j} \cdot y+\mathrm{k} \cdot z$.

Ebenso wie eine komplexe Zahl lässt sich eine Quaternion $q$ in Polardarstellung angeben [5]:

$q=|q| \mathrm{e}^{\mu \Phi}$.

Hierbei bezeichnet

$|q|=\sqrt{w^{2}+x^{2}+y^{2}+z^{2}}$

den Betrag von $q$, während $\mu$ eine Quaternion ohne Realteil, eine sogenannte reine Quaternion, ist. Diese wird berechnet durch

$\mu=\mathfrak{I}(q) /|\mathfrak{I}(q)|$.

Die Phase

$\Phi=\arccos (\Re(q) /|q|)$

stellt den Winkel zwischen Real- und Imaginärteil dar und lässt sich als ein Maß für den Anteil des Realteils an der gesamten Quaternion interpretieren.

Aufgabe von Verfahren zur Salienzbestimmung ist es, in Bildern diejenigen Bereiche auszumachen, die die Aufmerksamkeit eines menschlichen Betrachters auf sich ziehen. Dabei können Verfahren aus dem Stand der Technik nicht nur mit Grauwert-, sondern auch mit Farbbildern arbeiten. Auch die Verarbeitung von farbigen Bildserien (Videos) ist möglich. Verfahren wie jenes zur Bestimmung des Phasenspektrums der Quaternion-FourierTransformation (PQFT) [6] oder die Hyperkomplexe Fourier-Transformation (HFT) [7] weisen Kombinationen aus den drei Farbwerten eines Pixels den drei Imaginärteilen einer Quaternion zu. Ein optionaler Grauwertkanal, der im Fall von Bildfolgen die Änderung von einem Bild zum nächsten beschreibt, wird dem Realteil zugewiesen. Das so entstandene quaternionwertige Bild (jedes Pixel besteht aus einer Quaternion) wird der auf Quaternionen erweiterten zweidimensionalen QuaternionFourier-Transformation (QFT) unterzogen. Diese ermöglicht eine simultane Untersuchung aller Farbkanäle anstelle einer separaten Betrachtung der drei einzelnen Farbkanäle. Auf die genaue Vorgehensweise kann nicht eingegangen werden, es soll nur kurz angemerkt werden, dass analog zur reellen FourierTransformation auch das QFT-transformierte Bild Betrag und Phase besitzt. Es wird im Wesentlichen der Betrag manipuliert; bei PQFT wird er zu eins gesetzt, um nur die Phase zu berücksichtigen, während bei HFT das Betragsspektrum geglättet wird. Das Betragsquadrat des rücktransformierten Bilds ist direkt die Salienzkarte, die grauwertcodiert beschreibt, in welchen Bildregionen markante Objekte vorhanden sind. Markant in diesem Zusammenhang bedeutet, dass sie sich in Textur, Farbe oder ähnlichen Eigenschaften vom Hintergrund unterscheiden. Abbildung 2 zeigt beispielhaft die aus einem Farbbild berechnete Salienzkarte.
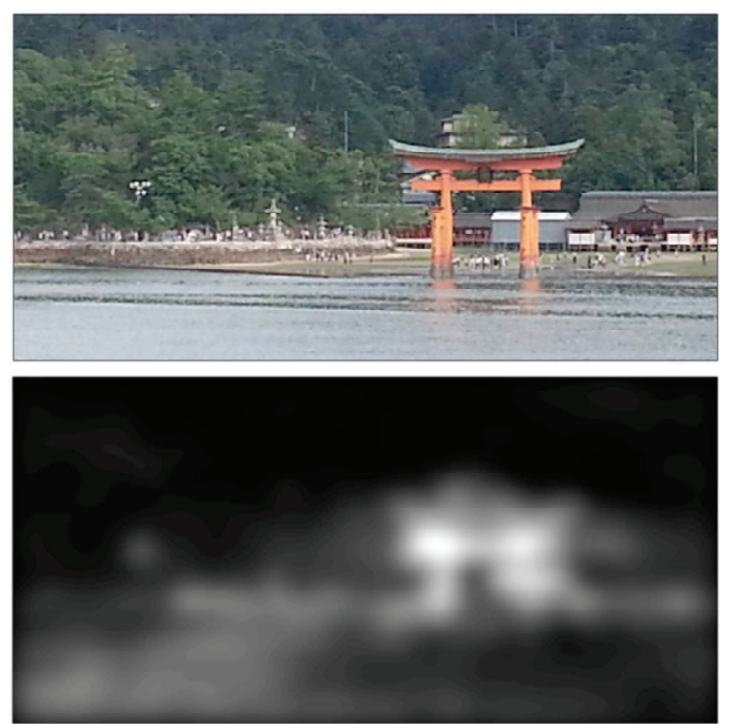

Abbildung 2: Beispiel für eine mit der HFTMethode [7] bestimmte Salienzkarte. 
Idee ist es nun, jeweils vier zweidimensionale Bilder aus dem vierdimensionalen Datensatz einem Quaternionenbild zuzuordnen. Von diesem wird eine Salienzkarte berechnet, die anzeigt, wie sich das Bild im Vergleich zu den drei anderen einbezogenen Bildern verhält (heller, dunkler, Änderung der Textur etc.). Durch Verschieben dieses Musters, wobei sukzessive neue Bilder mit einbezogen werden, ergeben sich neue Salienzkarten und zusammengefasst in spektraler Dimension betrachtet neue Spektren, die wir als Pseudospektren bezeichnen. Abbildung 3 und Abbildung 4 zeigen das Schema.

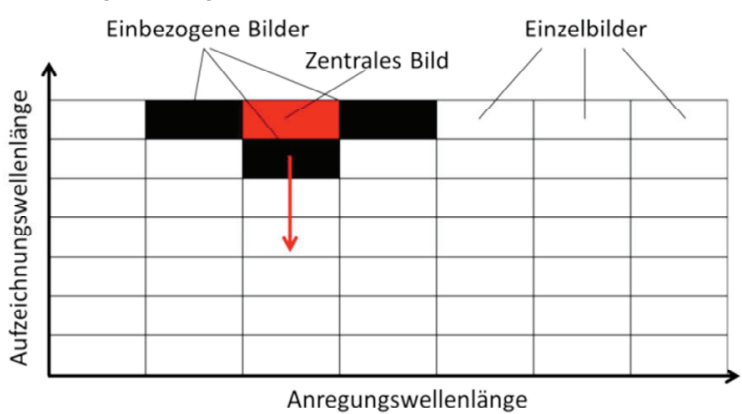

Abbildung 3: Schema des Einbezugs der Einzelbilder. Die Rechtecke stehen für zweidimensionale Einzelbilder der Szene bei fester Anregungs- und Aufzeichnungswellenlänge.

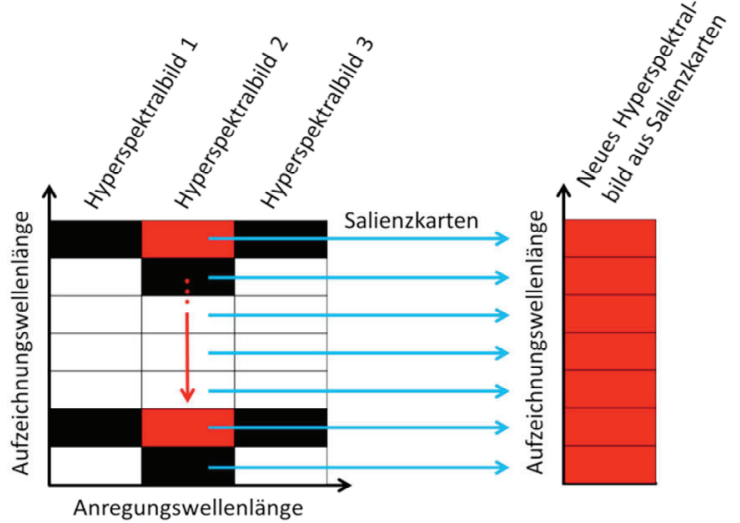

Abbildung 4: Zusammengesetzt aus den zweidimensionalen Salienzkarten ergibt sich ein neues Hyperspektralbild. Dessen Pixelspektren können als Pseudospektren angesehen werden.

\section{Ergebnisse der Salienzbestimmung}

Die Ergebnisse der Salienzbestimmung mit dem HFT-Verfahren sind in Abbildung 5 dargestellt. Es zeigt sich, dass das Verfahren sehr anfällig gegenüber kleinen salienten Bildregionen ist, aber große homogene Bereiche (also Bereiche der zweidimensionalen Einzelbilder, in denen keine große örtliche Variation vorliegt) in der Salienzkarte zu Werten nahe 0 gesetzt werden. Da homogene Bereiche, die zueinander unterschiedlich sind, dadurch in der Salienzkarte nicht mehr unterschieden werden können, eignet sich das HFTVerfahren nicht zur Fusion von mehreren dreidimensionalen Hyperspektralbildern, die bei verschiedenen Anregungswellenlängen aufgezeichnet wurden. Es ergeben sich innerhalb einer Probe teilweise Pseudospektren mit groBer Amplitude (in salienten Bildbereichen), teilweise aber auch Pseudospektren mit kleiner Amplitude (in homogenen Bildregionen). Wünschenswert ist allerdings, dass die Pseudospektren innerhalb einer Probe bzw. innerhalb einer Mineralsorte möglichst ähnlich sind und sich die Pseudospektren von verschiedenen Materialsorten möglichst deutlich unterscheiden.

Aus diesem Grund wird ein weiterer Ansatz untersucht, der zwar nicht die volle vierdimensionale Information berücksichtigt (für die Salienzbestimmung wird wie schon erwähnt die zweidimensionale QFT angewendet, die die örtliche Information innerhalb der zweidimensionalen Einzelbilder verarbeitet), aber dafür weniger Nichtlinearität aufweist.

Nachdem aus den vorliegenden Hyperspektralbildern vier zweidimensionale Bilder ausgewählt und aus innen ein quaternionwertiges Bild konstruiert wurde, lässt sich auch dieses quaternionwertige Bild direkt, also ohne Salienzbestimmung, weiterverwenden. Idee hierbei ist es, aus jeder Pixel-Quaternion genau einen Wert zu bestimmen, der dann diesem Pixel zugewiesen wird. Dazu wird auf die Polardarstellung einer Quaternion nach Gleichung (3) zurückgegriffen. Es lassen sich für diese der Betrag, die Achse und die Phase der Quaternion berechnen. Die Achse ist eine reine Quaternion $(w=0)$. Sie besteht somit aus drei Werten und kann nicht verwendet werden. Der Betrag besitzt nur einen Wert und lässt sich damit verwenden, hierbei ergibt sich allerdings der Nachteil, dass der Betrag proportional zu konstanten Faktoren in der Fluoreszenzintensität ist, d. h., wenn eine Stelle einer Probe weniger stark belichtet ist als eine andere, dann liegt die ausgesendete schwache Fluoreszenzintensität nicht an der schwachen Fluoreszenz des Minerals, sondern lediglich an der Beleuchtung. Dadurch wird also kein direkter Materialparameter bestimmt.

Die Phase einer Quaternion nach Gleichung (6) lässt sich umschreiben zu

$$
\Phi=\arccos \left(w / \sqrt{w^{2}+x^{2}+y^{2}+z^{2}}\right) \text {. }
$$

Somit geht in die Berechnung der Phase nicht nur der Betrag ein, sondern auch, wie groß der Realteil im Verhältnis zum Imaginärteil ist. Es wird also nur die relative Änderung zwischen Real- und Imaginärteil berücksichtigt; Belichtungsunterschiede spielen keine Rolle mehr. 

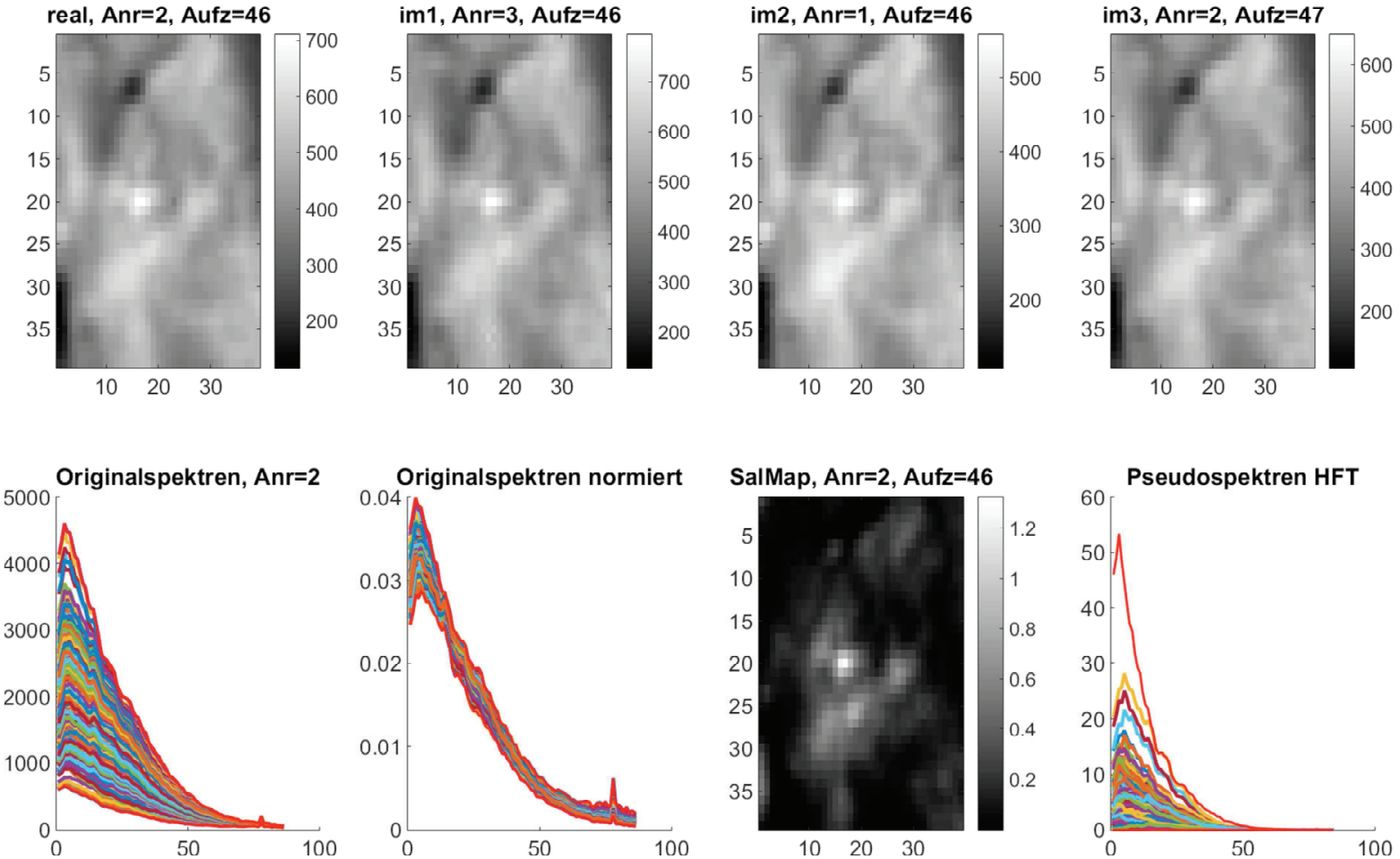

Abbildung 5: Ausschnitt der Größe 39x39 Pixel aus einem Bild einer Mineralprobe: Bestimmung der Salienzkarte (untere Reihe, zweites Bild von rechts) aus vier Einzelbildern (obere Reihe). Die Einzelbilder werden dem Realteil (real) bzw. den Imaginärteilen (im1-im3) einer Quaternion zugewiesen. Hierbei steht "Anr." für den Anregungskanal; 2 steht also für 300 nm Anregungswellenlänge. Analoges gilt für die Aufzeichnungswellenlänge („Aufz."). Die Salienzkarte betont lediglich kleine Bildregionen, wobei auch dunkle Regionen als salient erkannt werden (linke untere Ecke, um das 35. vertikale Pixel herum). Dadurch ändern sich zwar die Amplituden der Pseudospektren, aber nicht ihre Form.

Neben den Quaternionen gibt es auch Oktonionen, bei denen der Imaginärteil aus 7 Anteilen besteht. Bei diesen lässt sich die Phase dann analog zu Gleichung (6) bzw. (7) aus dem Arkuskosinus des Quotienten von Realteil und Betrag berechnen (siehe [8], Kap. 4). Abbildung 6 veranschaulicht die Bildung eines neuen Hyperspektralbilds bei Verwendung der 8 Hyperspektralbilder bei allen Anregungswellenlängen mittels Bildung der Phase. In Abbildung 7 sind die Pixelspektren bei $280 \mathrm{~nm}$ Anregungswellenlänge sowie die Pseudospektren, die sich bei Fusion der Hy-

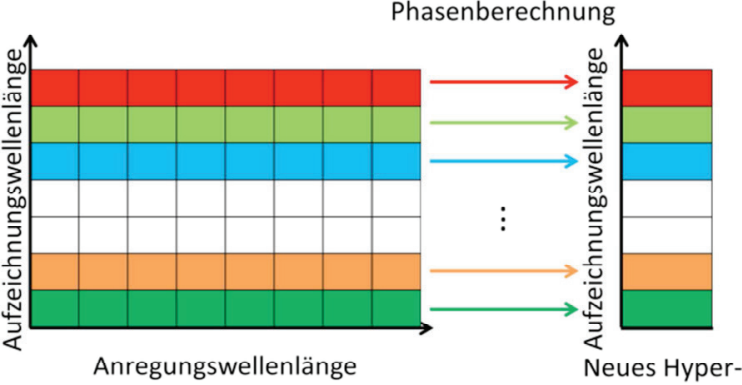

Abbildung 6: Erzeugung eines neuen Hyperspektralbilds aus 8 hyperspektralen Fluoreszenzbildern bei verschiedenen Anregungswellenlängen durch Berechnung der OktonionPhase. perspektralbilder bei allen 8 Wellenlängen mittels Berechnung der Oktonion-Phase ergeben, gezeigt. Obwohl die Originalspektren sehr ähnlich sind, ergeben sich in den Pseudospektren deutliche Unterschiede zwischen den verschiedenen Mineralsorten. Diese fusionierten Pixelspektren können dann für die Klassifikation der Pixel verwendet werden.

\section{Zusammenfassung und Ausblick}

Eine neuartige optische Bestimmungsmethode für Minerale wurde vorgestellt. Diese basiert auf der Ausnutzung von Fluoreszenzeffekten, wobei sich bei unterschiedlicher Anregungswellenlänge unterschiedliche Fluoreszenzspektren ergeben. Es wurde gezeigt, wie diese unterschiedlichen Fluoreszenzspektren einfach fusioniert werden können, sodass sich diskriminante Spektren ergeben. Der nächste Schritt besteht in der Klassifikation der Minerale mittels der ermittelten Pseudospektren. 

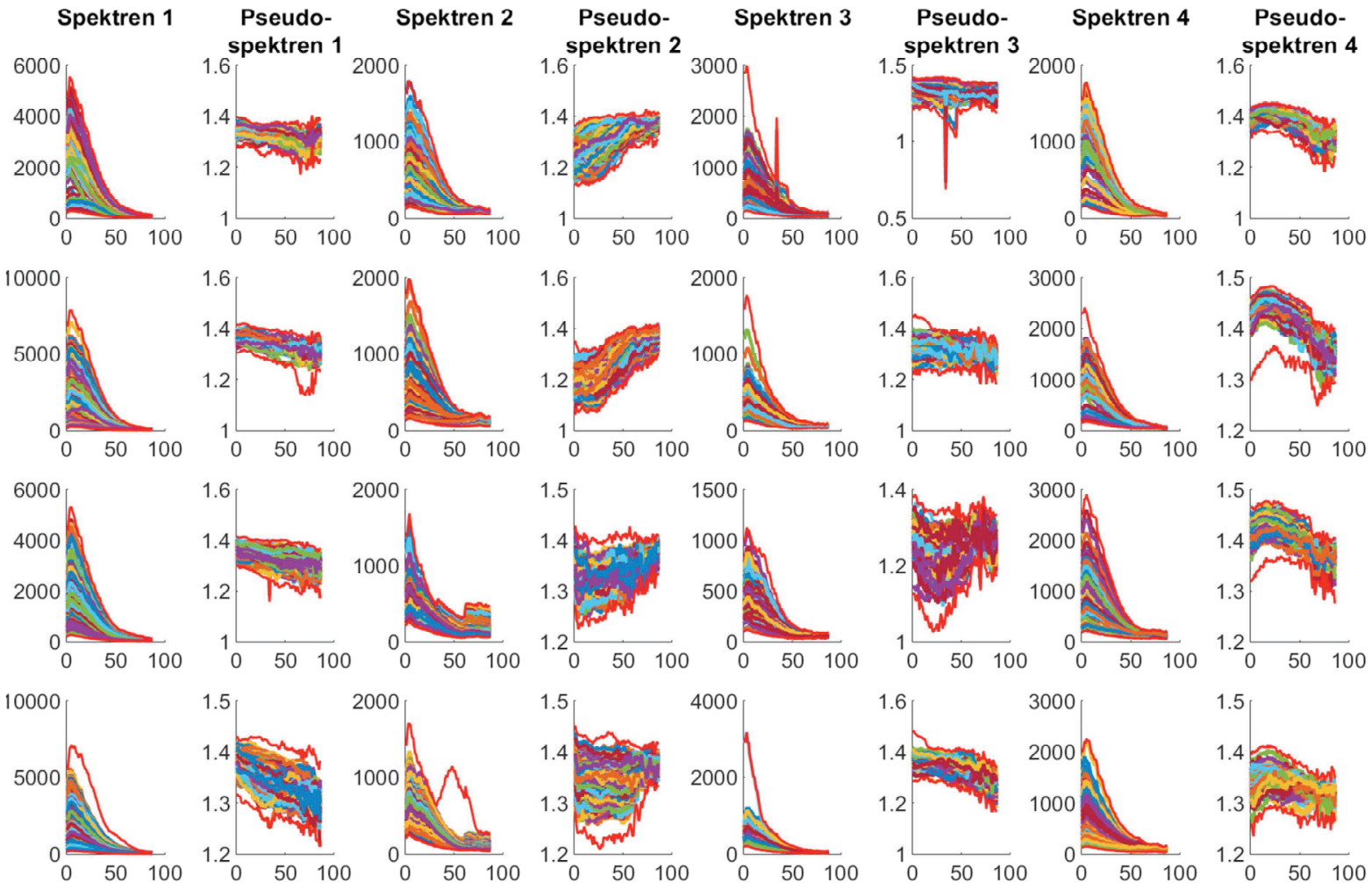

Abbildung 7: Spektren (Anregungswellenlänge $280 \mathrm{~nm}$ ) und Pseudospektren (erzeugt mittels Oktonionen aus allen 8 vorliegenden Hyperspektralbildern) von jeweils vier Proben der ersten vier Mineralsorten. Spaltenweise werden zunächst die Spektren der ersten vier Proben der ersten Sorte, dann deren Pseudospektren, danach die Spektren der ersten vier Proben der zweiten Sorte usw. angezeigt. Es fällt auf, dass, obwohl die Form der Spektren bei allen Mineralen sehr ähnlich ist, die Pseudospektren sich zwischen den Sorten deutlicher unterscheiden. Die Pseudospektren der ersten Sorte haben einen leicht abfallenden Verlauf, die der zweiten einen ansteigenden, die der dritten einen relativ konstanten und die der vierten einen erst ansteigenden, dann wieder abfallenden Verlauf.

\section{Literaturnachweis}

[1] T. Horrocks, D. Wedge , E.-J. Holden, P. Kovesi, N. Clarke und J. Vann, "Classification of GoldBearing Particles Using Visual Cues and CostSensitive Machine Learning," Mathematical Geosciences, Bd. 47, Nr. 5, pp. 1-25, Juli 2015. doi: 10.1007/s11004-015-9597-7

[2] S. Bauer und F. Puente León, „Gewinnung und Verarbeitung hyperspektraler Fluoreszenzbilder zur optischen Mineralklassifikation," tm-

Technisches Messen, Bd. 82, Nr. 1, pp. 24-33, 2015. doi: 10.1515/teme-2014-0018

[3] S. Bauer und F. Puente León, „Spectral and geometric aspects of mineral identification by means of hyperspectral fluorescence imaging," tm - Technisches Messen, Bd. 82, 2015. doi: 10.1515/teme-2015-0039

[4] M. Gaft, R. Reisfeld und G. Panczer, Modern luminescence spectroscopy of minerals and materials, Springer, 2015.

[5] S. Sangwine und T. Ell, "Hypercomplex autoand cross-correlation of color images," Proceedings of the International Conference on Image Processing, 1999, Bd. 4, pp. 319-322, 1999. doi: 10.1109/ICIP.1999.819603

[6] C. Guo, Q. Ma und L. Zhang, „Spatio-temporal saliency detection using phase spectrum of qua- ternion Fourier transform," IEEE Conference on Computer Vision and Pattern recognition

(CVPR), pp. 1-8, 2008. doi:

10.1109/CVPR.2008.4587715

[7] J. Li, M. Levine, X. An, X. Xu und H. He, „Visual saliency based on scale-space analysis in the frequency domain," IEEE Transactions on Pattern Analysis and Machine Intelligence, Bd. 35, Nr. 4, pp. 996-1010, 2013. doi: 10.1109/TPAMI.2012.147

[8] T. Dray und C. Manogue, The Geometry of the Octonions, World Scientific Publishing, Singapur, 2015. 